\title{
Lehre und Forschung
}

\author{
Frederic Hanusch
}

\section{Zur Feindschaft zwischen Erkenntnisfortschritt und Plagiarismus}

„Stanford University said today it had learned that its teaching assistants ' handbook section on plagiarism had been plagiarised by the University of Oregon. Stanford issued a release saying Oregon officials conceded that the plagiarism section and other parts of its handbook were identical with the Stanford guidebook. Oregon officials apologised and said they would revise their guidebook.“ (New York Times 1980: A28)

\section{Die Debatte um Plagiatsaufdeckungen und ihre Folgen}

Wissenschaft wird um des Erkenntnisfortschritts Willen betrieben, sie ist, wie es der Duden ausdrückt, eine „Wissen hervorbringende forschende Tätigkeit“, zu der Promovierende beitragen (Duden 2014 a). Dies dürfte all jene überraschen, die nicht selbst im Wissenschaftsbetrieb tätig sind und denen er im Zuge der Plagiatsaufdeckungen der vergangenen Jahre eher vorgekommen sein mag wie ein Karrierekatalysator für aufstrebende Politikerinnen und Politiker, bei dem es vor allem darauf ankommt, möglichst viele Zitate mittels Anführungszeichen und Fußnoten mit höchster Präzision aneinanderzureihen. Weder davon, was Wissenschaft eigentlich auszeichnet, noch von den durch die nichtintendierten Folgen der Plagiatsaufdeckungen betroffenen gegenwärtigen und zukünftigen Promovierenden war bisher die Rede. Das kann und wird sich ändern, zumindest lassen dies erste Initiativen und Forschungsprojekte vermuten. ${ }^{1}$ Dabei geht es nicht nur, wie es vordergründig erscheint, um ein paar Gänsefüßchen, sondern vielmehr um das Verständnis darüber, was Wissenschaft ausmacht und das heißt in der konkreten Forschungspraxis

1 Siehe beispielsweise die am Kulturwissenschaftlichen Institut Essen (KWI) entstandenen „Essener Thesen“, die im folgenden Text Eingang finden (KWI 2014), oder das Forschungsprojekt ,Zitat und Paraphrase" der Berlin-Brandenburgischen Akademie der Wissenschaften (BBAW 2014). Eine wissenschaftliche Beschäftigung mit Plagiarismus steht in vielen Teilbereichen noch aus (s. u.). 
der Dissertation nicht (nur) die Beseitigung von Altlasten, sondern um Perspektiven und Lehren aus der Debatte für gegenwärtige und zukünftige Promovierende.

Welche Lehren sind also zum Wohl der Wissenschaft und Promovierender aus den (tatsächlichen und vermeintlichen) Plagiatsaufdeckungen zu ziehen? Zur Beantwortung dieser Frage erfolgt zunächst eine grundlegende Klärung, was eine Dissertation und was ein Plagiat ausmachen. Sodann werden erste Handlungsfelder identifiziert sowie exemplarisch in einem Handlungsfeld eine Teillösung angedeutet. Abschließend werden dreierlei Lehren skizziert: Erstens ist der Schlüssel zum Erkenntnisfortschritt und damit zur Verhinderung von Plagiarismus in einer originellen Fragestellung zu finden; zweitens bestehen wissenschaftsinterne Lösungsansätze, problematischer erscheinen jedoch wissenschaftsexterne Herausforderungen; drittens existieren zu Ausmaß, Deutung und Ursachen von Plagiarismus umfangreiche Desiderata.

\section{Was zeichnet eine Dissertation und was ein Plagiat aus?}

Möchte man über Plagiarismus und Dissertationen schreiben, müssen diese in einen Bezug zueinander gesetzt werden, andernfalls lässt sich das Problem nicht identifizieren und keine Lehren daraus ziehen. Der einleitend genannten Definition folgend, dass es sich bei der Wissenschaft um eine „Wissen hervorbringende forschende Tätigkeit" handelt, ist die Dissertation als ein Teil derselben zu sehen. Dabei arbeiten Promovierende wie alle Wissenschaftler zwar eigenverantwortlich, stehen aber zugleich auf den „Schultern von Riesen“ (Merton 1983), sind also Teil einer auf kollektiver Arbeit fußenden Wissensproduktion.

Diese Auffassung findet sich auch im deutschen Urheberrechtsgesetz wieder, das nur die äußere Formgebung wissenschaftlicher Werke, nicht aber Ideen und Erkenntnisse unter Schutz stellt (Weiblinger 2012: 43). Hierbei handelt es sich um eine Auffassung, die insbesondere seit der Aufklärung an Bedeutung gewonnen hat: Bereits Fichte unterschied zwischen Materiellem, ,den Inhalt des Buchs, die Gedanken die es vorträgt“, und Form, also „die Art wie, die Verbindung in welcher, die Wendungen und die Worte, mit denen es [das Buch] sie vorträgt", wobei das Materielle mit Publikation frei werde, die Form aber Eigentum bleibe (Fichte 2014; Weiblinger 2012: 58). Konsequenterweise wird von der Schutzunfähigkeit des Inhalts gesprochen, denn nur dadurch, dass bereits gewonnene Erkenntnisse zur Verfügung stehen, ist wissenschaftlicher Fortschritt möglich (ebd.: 60). Wohlwissend, dass nicht jede Erkenntnis neu sein kann, ist die Dissertation auf eine originelle Fragestellung angewiesen, die bis zu diesem Zeitpunkt noch gar nicht oder nach- 
weislich falsch oder unvollständig beantwortet wurde, um zum Erkenntnisfortschritt der kollektiven Wissensproduktion beizutragen.

Auch das Plagiat besitzt eine lange Geschichte und nicht immer war es so negativ konnotiert wie gegenwärtig, zuweilen war es gar ein Sport unter Gelehrten, fremde Ideen in ihre Arbeiten einzubauen, die von anderen erkannt werden sollten (Theisohn 2009). Doch was ist ein Plagiat? Allgemein wird als Plagiat die ,unrechtmäßige Aneignung von Gedanken, Ideen o. Ä. eines anderen auf künstlerischem oder wissenschaftlichem Gebiet und ihre Veröffentlichung“ und damit der „Diebstahl geistigen Eigentums" verstanden (Duden 2014 b). Präziser gefasst und auf den Bereich der Wissenschaft fokussiert, kann von einem Plagiat gesprochen werden, wenn jemand

- „uses words, ideas, or work products

- attributable to another identifiable person or source

- without attributing the work to the source from which it was obtained

- in a situation in which there is a legitimate expectation of original authorship

- in order to obtain some benefit, credit, or gain which need not be monetary" (Fishman 2009: 5).

Inwiefern Intentionalität bei der Beurteilung eines Plagiats eine Rolle spielt, ist nicht abschließend diskutiert. ${ }^{2}$ Obgleich es sich hierbei um eine nur schwer zu ermittelnde Größe handeln dürfte, darf sie nicht unberücksichtigt bleiben, denn die Verurteilung aufgrund des Nichtwissens um eine bereits an anderer Stelle formulierte Idee, Wortreihungen etc. erscheint zumindest fragwürdig.

Doch wie groß ist das Problem des Plagiarismus? Hinsichtlich der Größenordnung existieren zumindest für Deutschland kaum belastbare Zahlen, weshalb ein internationaler Vergleich aufgrund fehlender Zahlen nahezu unmöglich ist (Hornbostel 2012: 7 f.; Hauss u. a. 2012: 19; Weber-Wulff 2014: 19 f.). ${ }^{3}$ Letztlich ist auch unklar, ob die Anzahl öffentlich gewordener Fälle tatsächlich darauf hindeutet, dass Plagiarismus zunimmt oder ob beispielsweise lediglich besser kontrolliert wird. ${ }^{4}$ Zudem stehen Studien aus, die erörtern, inwieweit es sich um ein kulturspezifisches Phänomen handelt, also welche Wissenschaftstraditionen gegeben sind (Bosbach 2012; Bruckner 2013). Auch lassen sich keine belastbaren Zahlen finden, inwiefern der Plagiarismus ein sich ausweitendes Problem darstellt, jedoch kann davon aus-

2 So reißt Fishman das Thema zwar kurz an (Fishman 2009: 2), in der Definition wird aber nicht mehr darauf eingegangen, inwiefern es sich um einen intentionalen Akt handeln muss.

3 Eine erste Bestandsaufnahme der Promovierenden umfassender Art unternimmt das Institut für Forschungsinformation und Qualitätssicherung (iFQ) mit dem Promovierendenpanel ProFile (http:// www.forschungsinfo.de/profile/start.html).

4 A-Drs. 17(18)211 b, 3. 
gegangen werden, dass das Auftreten des Internets Plagiarismus technisch vereinfacht, gleichsam aber auch leichter auffindbar gemacht hat.

Fest steht im Sinne der Originalität: „Der Plagiator stellt nichts her, er kaschiert nur seine Kopistenarbeit.“ (Leggewie/Mühlleitner 2007: 206 f.) Sofern also - und dieser Punkt scheint von zentraler Bedeutung zu sein - eine originelle Fragestellung vorliegt, können weder Ideen noch Arbeitsergebnisse und nur in bedingtem Maße Wörter oder Wortreihenfolgen (beispielsweise im Literaturüberblick) plagiiert werden, das Materielle im Sinne Fichtes wird somit nahezu unmöglich plagiierbar.

\section{Handlungsfelder, Herausforderungen, mögliche Lösungen}

Plagiate sind schlimm, da sie das Wissenschaftssystem und den Plagiierten schädigen sowie den Erkenntnisfortschritt behindern. Ein einfacher Denkschritt dergestalt, dass mit der Bearbeitung und Zurschaustellung von Fehlverhalten den Wissenschaftlern das Plagiieren schon auszutreiben sei, greift allerdings zu kurz, das Phänomen ist komplexer. Nach der generellen Einführung und Fokussierung der Problemlage soll es daher nun darum gehen, die in diesem Kontext sechs bedeutendsten Handlungsfelder zu skizzieren, die, so die These, Einfluss auf Plagiarismus besitzen und positiv gewendet Zukunftsperspektiven aufzuzeigen vermögen. Hierzu erscheint eine tabellarische Übersicht sinnvoll (Tab. 1), die das Handlungsfeld, die Herausforderungen und mögliche (Teil-)Lösungen überblicksartig aufspannen, ohne dass auf alle Aspekte eingegangen werden kann.

\section{Die Motivation zur Promotion}

Die sich aus der Tabelle ergebenden Handlungsmöglichkeiten werden nun anhand einer Teillösung in einem der Handlungsfelder, der Motivation zur Promotion, illustriert. Die konkrete Herausforderung bilden in diesem Fall wissenschaftsexterne Anreize eine Dissertation anzufertigen.

Zwar stellt die Promotion eine wissenschaftliche Weiterqualifizierung dar, jedoch dient sie auch als repräsentatives Kapital (Best 2012: $33 \mathrm{f}$.) und als (Ersatz-)Indikator für die Befähigung eines Bewerbers, womit der Doktortitel ein Talentsignal in der außerwissenschaftlichen Welt bildet (Franck 2005: 6). Hierdurch erhöht sich der Anreiz für Dissertationen, deren Verfasser ihre Motivation nicht im Erkenntnisfortschritt verorten. Diese Sorte von Dissertation wird ihres originären Zwecks entfremdet, sie soll somit nicht mehr Referenzpunkt im Wissenschaftssystem sein und zur Anerkennung erzeugten Erkenntnisfortschritts in ebendiesem führen, sondern als Hebel zum wissenschaftsexternen Karrieresprung dienen. Hiermit 


\section{Tabelle 1: Übersicht zu Handlungsfeldern, Herausforderungen und möglichen Lösungen}

\begin{tabular}{|c|c|c|}
\hline Handlungsfeld & Herausforderung & Mögliche Lösung \\
\hline $\begin{array}{l}\text { Motivation eine Promotion anzu- } \\
\text { fertigen }\end{array}$ & $\begin{array}{l}\text { Doktortitel begünstigt außerwis- } \\
\text { senschaftliche Karriere, Möglich- } \\
\text { keit des käuflichen Erwerbs von } \\
\text { Ehrendoktoren }\end{array}$ & $\begin{array}{l}\text { Wissenschaftsexterne Anreize mi- } \\
\text { nimieren, (institutionalisierte) } \\
\text { Überprüfung der Motivation bei } \\
\text { Annahme von Doktoranden, Wie- } \\
\text { dereinbettung des Doktortitels als } \\
\text { wissenschaftliche Weiterqualifi- } \\
\text { zierung, Promotionsrecht bei Uni- } \\
\text { versitäten belassen }\end{array}$ \\
\hline $\begin{array}{l}\text { Zitationsregeln und Plagiatsvor- } \\
\text { schriften }\end{array}$ & $\begin{array}{l}\text { (Fehlende Kenntnis über) fachspe- } \\
\text { zifische Konventionen, uneinheit- } \\
\text { liche Zitationsregeln }\end{array}$ & $\begin{array}{l}\text { Liberale (d. h. aufzeigen wie es } \\
\text { nicht sein darf ohne zu diktieren } \\
\text { wie es sein muss) Vereinheitli- } \\
\text { chung von Plagiatsvorschriften } \\
\text { nach (inter)disziplinären Stan- } \\
\text { dards }\end{array}$ \\
\hline $\begin{array}{l}\text { Zuständigkeit (öffentlich) Ver- } \\
\text { dacht zu äußern und Anklage zu } \\
\text { erheben }\end{array}$ & $\begin{array}{l}\text { Legitimationsbasis für Einzelper- } \\
\text { sonen (mit wirtschaftlichen Inte- } \\
\text { ressen wie Kamenz, }{ }^{6} \text { Heidingsfel- } \\
\text { der) }{ }^{7} \text { und Wikis (wie Guttenplag, } \\
\text { Vroniplag) gegenüber institutio- } \\
\text { nalisierter Wissenschaft, mögli- } \\
\text { cher Rufmord }\end{array}$ & $\begin{array}{l}\text { Rückführung jeglicher Verdachts- } \\
\text { momente in den institutionalisier- } \\
\text { ten Wissenschaftsbetrieb mittels } \\
\text { gestärkten Ombudswesens und } \\
\text { klaren Verfahrensregeln wie von } \\
\text { DFG vorgeschlagen, Prüfung zen- } \\
\text { traler Anlaufstelle }\end{array}$ \\
\hline $\begin{array}{l}\text { (Nichtintendierte) Folgen der Pla- } \\
\text { giatsaufdeckungen }\end{array}$ & $\begin{array}{l}\text { Starke Verunsicherung }{ }^{8} \text { unter Pro- } \\
\text { movierenden führt dazu, dass im- } \\
\text { mer mehr Ressourcen in mehrfa- } \\
\text { che Überprüfung eines Zitats statt } \\
\text { in Mehrung origineller Erkenntnis } \\
\text { investiert wird }\end{array}$ & $\begin{array}{l}\text { Pflege und Akkuratesse von Zita- } \\
\text { tion muss dem Erkenntnisfort- } \\
\text { schritt dienen, Betreuer hat dies zu } \\
\text { vermitteln }\end{array}$ \\
\hline Auswertungsmethoden & $\begin{array}{l}\text { Mathematisierung von Text durch } \\
\text { Software aber auch manuell, Text } \\
\text { wird nicht gedeutet sondern ver- } \\
\text { wendet und in Barcodes, Prozent- } \\
\text { zahlen etc. aufbereitet }\end{array}$ & $\begin{array}{l}\text { Ablehnung solcher Verfahren, } \\
\text { Anwendung bzw. Entwicklung } \\
\text { adäquater und zugleich vermittel- } \\
\text { barer Alternativen }\end{array}$ \\
\hline Strukturelle Gegebenheiten & $\begin{array}{l}\text { Publikationsdruck (publish or pe- } \\
\text { rish), Prekarisierung insbesondere } \\
\text { bei Individualpromotion, Multi- } \\
\text { funktion Doktorvater/-mutter (be- } \\
\text { treuend, vorgesetzt, prüfend) kann } \\
\text { positiv wie negativ sein }\end{array}$ & $\begin{array}{l}\text { Beispiel Schweden: in der Regel } \\
\text { fünfjährige Festanstellung nach } \\
\text { Aufnahmeverfahren, dabei Über- } \\
\text { nahme der Wissenschaftsnormen } \\
\text { wahrscheinlicher als bei prekärer } \\
\text { Individualpromotion, erste Publi- } \\
\text { kationen }\end{array}$ \\
\hline
\end{tabular}

Quelle: Eigene Zusammenstellung.

5 Aufschlussreich hinsichtlich der unterschiedlichen Positionen ist in diesem Zusammenhang die auf VroniPlag geführte Debatte über Zitationsregeln in Jura: http://de.vroniplag.wikia.com/index.php? title=Forum:Spezielle_Zitierweise_bei_Juristen\%3F\&t=20110821220429 (Stand: 29.5.2014). 
ist selbstverständlich weder gesagt, dass alle Dissertationen, deren Anfertigung auf einer Motivation zum Erkenntnisfortschritt fußen, frei von Plagiaten und voll von Originalität sind, noch, dass alle anderen Dissertationen gleichsam plagiiert und frei von Originalität seien. Dennoch sei hier die These aufgestellt, dass sich eine entsprechende Verbindung finden lässt, zumindest deutet ein Anfangsverdacht durch die bekannt gewordenen Plagiatsfälle der vergangenen Jahre darauf hin.

Abbildung 1: Anteil der Akademiker und Promovierten im deutschen Nationalparlament seit 1848

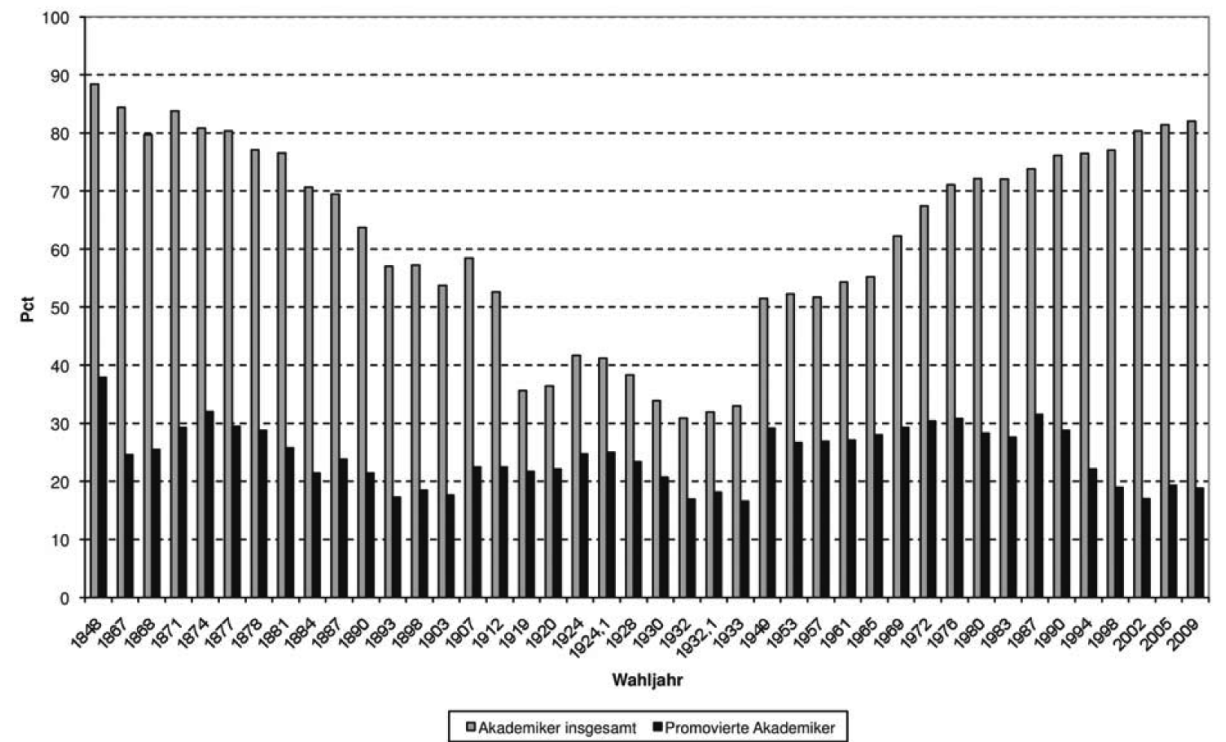

Quelle: Best 2012: 34.

6 Uwe Kamenz betreibt „ProfNet“ (www.profnet.de) und ist insbesondere durch den Fall Steinmeier einer größeren Öffentlichkeit bekannt geworden, da der vom Focus in Auftrag gegebene und von Kamenz angefertigte Prüfbericht häufig aufgrund der hohen „Gesamtplagiatswahrscheinlichkeit“ zitiert wurde (Spiegel Online 2013).

7 Besonders anschaulich in einer Art „Kopfgeldtabelle“ von Martin Heidingsfelder zu sehen: http:// politplag.de/index.php/Kandidatenliste (Stand: 29.5.2014).

8 Diese Verunsicherung bei verängstigten, aber gewissenhaft arbeitenden Promovierenden schlägt sich auch im VroniPlag-Forum nieder: „Es rufen aber in der Tat ab und zu Leute bei mir an und wollen, dass VroniPlag Wiki ihre Doktorarbeiten 'vorab' prüft. Sie glauben, es gibt eine VroniPlag Wiki-Zauber-Plagiatserkennungs-Software (tm), man drückt auf dem Knopf und ein Urteil wird gefällt. Ich antworte immer: Sprechen Sie mit Ihrem Betreuer. So eine Software gibt es nicht" (http:// de.vroniplag.wikia.com/wiki/Diskussionsfaden:31053; Stand: 29.5.2014). 
Erste Anzeichen für außerwissenschaftlichen Reputationsgewinn lassen sich bei promovierten Abgeordneten finden, die seit 1848 im Verhältnis zur Durchschnittsbevölkerung weitaus häufiger im Parlament vertreten waren (Abb. 1).

Obgleich Promovierte mit höherer Wahrscheinlichkeit ein Direktmandat sowie einen besseren Listenplatz erreichen (Manow/Flemming 2011) und der Anteil Promovierter deutlich über dem Bevölkerungsdurchschnitt liegt, ist bei den letzten Wahlen ein Rückgang dieses Anteils zu verzeichnen. Dieser ist möglicherweise zurückzuführen auf den bereits stattfindenden Verlust des Doktortitels als Distinktionsmerkmal oder auch auf den immer gefüllteren Terminkalender von Berufspolitikern und die fehlende Zeit, um eine Promotion anzufertigen, wodurch das Distinktionsmerkmal langfristig auch wieder an Bedeutung gewinnen könnte (Best 2012: 34 ff.).

Vergleicht man die Anzahl promovierter Politiker jedoch mit Führungspersonen in der Wirtschaft, lässt sich eine relativ größere Zahl Promovierter finden. So ergab eine Umfrage unter 61 Topmanagern in 2007, dass 50\% promoviert waren, wobei dieser Umstand bei den DAX 30 Unternehmen mit 20 von 30 promovierten Managern besonders ausgeprägt war (Buß 2012: $25 \mathrm{ff}$.).

Vergleicht man nun Wirtschaftseliten und politische Eliten europaweit, zeigt sich, dass Wirtschaftseliten in der BRD ungewöhnlich häufig promoviert sind (Abb. 2). Der Doktortitel ist somit ein klarer Hebel zum Aufstieg in die politische Elite, insbesondere innerhalb der Wirtschaftselite. Zudem ist erkennbar, dass die Wertigkeit des Doktortitels gänzlich unterschiedlich ausfällt. ${ }^{9}$

Wie kann diese Herausforderung angegangen werden? Es gilt Wege zu finden, wie der außerwissenschaftlichen Überhöhung des Doktortitels als Distinktionsmerkmal begegnet werden kann. Hierzu gilt es zum einen entsprechende Substitute als Talentsignal zu finden, wie dies der MBA im Bereich der Wirtschaft zunehmend sein kann, aber es gilt auch den Doktorgrad wieder als wissenschaftliches Qualifikationsmerkmal zu definieren und kenntlich zu machen, als das er originär dient und nicht außerwissenschaftlicher Reputationsverschaffung. Eine erste konkrete Maßnahme ist in diesem Zusammenhang die Abschaffung der Eintragung des Doktortitels in Ausweisen (Personalausweis, Reisepass).

In den letzten beiden Legislaturperioden (16 und 17) gab es Bestrebungen, den Doktortitel aus dem Pass zu streichen. Eine erste Initiative der Bundesregierung

9 Hierbei sind mögliche Unterschiede zu beachten, wie jene, dass in Großbritannien der Ort, an dem ein akademischer Abschluss erworben wurde, ein höheres Gewicht besitzt (Oxford, Cambridge) und in einigen osteuropäischen Staaten ein kleines Doktorat existiert (Best 2012: 40). 
Frederic Hanusch

Abbildung 2: Anteil promovierter Wirtschaftseliten und politischer Eliten im europäischen Vergleich

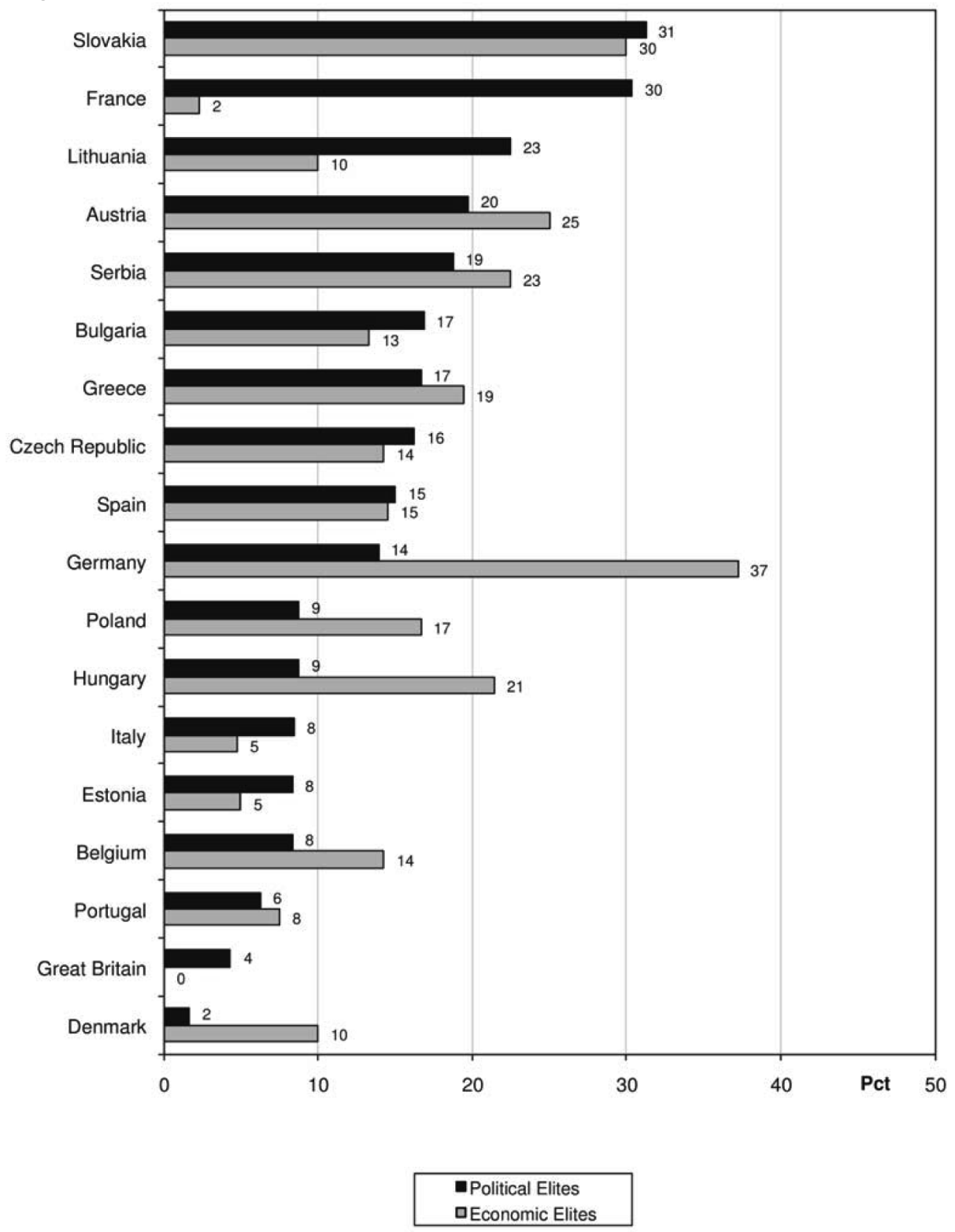

Quelle: Best 2012: 39. 
unternahm dazu den Versuch unter Federführung des Innenministeriums 2007. ${ }^{10}$ Die Abschaffung des Doktortitels sollte im Zuge der Änderungen des Passgesetzes erfolgen. Begründet wurde dies unter anderem mit Schwierigkeiten bei der Anerkennung von ausländischen Doktorgraden im Zuge der Passausstellung, da die ausstellenden Behörden oftmals nicht über die hinreichende Sachkenntnis verfügten. ${ }^{11}$ Darüber hinaus entspreche der Doktortitel in Ausweisen auch nicht den internationalen Gepflogenheiten. ${ }^{12}$ Die Abschaffung scheiterte jedoch noch im selben Jahr im Bundesrat. ${ }^{13}$ Insbesondere Bayern und Thüringen sprachen sich für eine Beibehaltung aus „,Rücksicht auf die deutschsprachige Kulturtradition“ und ,jahrzehntelange Praxis" aus. ${ }^{14}$ Einen erneuten Versuch zur Abschaffung unternahm 2011 die Fraktion Bündnis 90/Die Grünen. ${ }^{15}$ Im Gesetzesentwurf werden eine Reihe von Gründen angeführt, unter anderem dass der Doktortitel kein Bestandteil des Namens sei (andernfalls müssten auch andere Qualifizierungsgrade wie Magister oder Meister eingetragen werden können), es sich um einen wissenschaftlichen Qualifizierungsnachweis handele und nicht um eine ehrenvolle Anerkennung, die Eintragung weder in der Regelung der EU zur einheitlichen Passgestaltung noch im internationalen Standard (Doc 9303) vorgesehen sei, Behörden entlastet würden, keine Kosten entstünden und der „Dr.“ zur Identifizierung einer Person nicht nötig sei. ${ }^{16}$ Der Gesetzesentwurf wurde mit Stimmen der CDU/CSU und FDP gegen die Stimmen von SPD, Die Linke und Bündnis 90/Die Grünen abgelehnt. ${ }^{17}$

Sowohl das Scheitern 2007 als auch 2011 ist aus Sicht der Wissenschaft und insbesondere der Eindämmung von Plagiaten als bedauernswert zu bezeichnen, bot sich doch die Chance, der außerwissenschaftlichen Überhöhung des Doktortitels Einhalt zu gebieten.

\section{Vorläufige Lehren und Ausblick}

Drei vorläufige Lehren können aus den vorangegangenen Ausführungen gezogen werden:

1. Zentral zur Verhinderung von Plagiarismus (in Dissertationen) ist eine originelle Fragestellung, die dem Erkenntnisfortschritt dient. Es sollte daher stets im Blick

10 BT-Drs. 16/4138.

11 BT-Drs. 16/4138, 14.

12 BT-Drs. 16/4138, 14.

13 BT-Drs. 16/5445.

14 BT-Drs. 16/5445, 11.

15 BT-Drs. 17/8128.

16 BT-Drs. 17/8128, $5 \mathrm{f}$.

17 BT-Drs. 17/11908. 
behalten werden, Promovierende des Erkenntnisfortschritts zu befähigen anstatt darauf zu zielen ihnen das Plagiieren auszutreiben; hier bedingt Ersteres das Ausbleiben von Letzterem und ist der direkteste Weg zur Anerkennung von Wissenschaftsnormen.

2. Lösungen sind zwar auch in der Stärkung wissenschaftsinterner Verfahren zu sehen, zuvorderst scheinen jedoch die wissenschaftsexternen Herausforderungen einer gründlichen Auseinandersetzung zu bedürfen.

3. Es gibt für die BRD keine belastbaren Zahlen zu Ursachen, Deutung und Ausmaß von Plagiarismus, ebenso wenig ob es sich um ein zunehmendes, gleichbleibendes oder abnehmendes Phänomen handelt. Zudem erscheinen zivilgesellschaftliche Plattformen wie GuttenPlag oder VroniPlag ein interessantes Forschungsobjekt darzustellen, deren Wissenschaftsverständnis sowie genauen Funktions- und Wirkungsweisen bisher noch wenig untersucht wurden.

Obgleich Plagiate vermutlich niemals vollständig verschwinden werden, gibt es ermutigende Signale, dass die Wissenschaft gestärkt aus der Debatte um Plagiatsaufdeckungen hervorgehen kann. Interne Verfahrensregeln wurden und werden wie in der Denkschrift zur "Sicherung guter wissenschaftlicher Praxis“ der Deutschen Forschungsgemeinschaft (DFG) oder in der Empfehlung „Gute wissenschaftliche Praxis an deutschen Hochschulen“ der Hochschulrektorenkonferenz (HRK) bestätigt und präzisiert (DFG 2013; HRK 2013). Problembehafteter erscheinen wissenschaftsexterne Herausforderungen, sei es die skizzierte Abschaffung des Doktortitels oder die medial zuhauf replizierte Mathematisierung von Text. Hier kann die institutionalisierte Wissenschaft den Dialog suchen und Alternativen aufzeigen, damit sie sich in Bälde wieder ganz der Schaffung von Erkenntnisfortschritt widmen kann.

\section{Literatur}

$B B A W, 2014:$ Zitat und Paraphrase, http://www.bbaw.de/forschung/zitat-und-para phrase/uebersicht (Stand: 22.5.2014).

Best, Heinrich, 2012: Promotionen von Politikern im historischen und internationalen Vergleich, in: Nathalie Huber/Anna Schelling/Stefan Hornbostel (Hrsg.), Der Doktortitel zwischen Status und Qualifikation, iFQ-Working Paper No. 12, November 2012, 33-42, http://www.forschungsinfo.de/Publikationen/Down load/working_paper_12_2012.pdf (Stand: 23.5.2014).

Bosbach, Eva, 2012: „And how do I fly again, exactly?" Qualitätssicherung in der geisteswissenschaftlichen Doktorandenausbildung durch Institutionalisierung der Individualpromotion - Beispiele aus Deutschland und den USA, in: Nathalie 
Huber/Anna Schelling/Stefan Hornbostel (Hrsg.), Der Doktortitel zwischen Status und Qualifikation, iFQ-Working Paper No. 12, November 2012, 125-132, http://www.forschungsinfo.de/Publikationen/Download/working_paper_12_2 012.pdf (Stand: 23.5.2014).

Bruckner, Johanna, 2013: Lieber ein Plagiatsfall als eine Sexaffäre, http://www.su eddeutsche.de/bildung/wissenschaftsskandale-von-us-politikern-lieber-ein-pla giatsfall-als-eine-sexaffaere-1.1593833 (Stand: 23.5.2014).

Buß, Eugen, 2012: Die Akademisierung der Vorstandsetagen, in: Nathalie Huber/ Anna Schelling/Stefan Hornbostel (Hrsg.), Der Doktortitel zwischen Status und Qualifikation, iFQ-Working Paper No. 12, November 2012, 25-31, http://www .forschungsinfo.de/Publikationen/Download/working_paper_12_2012.pdf (Stand: 23.5.2014).

$D F G$, 2013: Sicherung guter Wissenschaftlicher Praxis. Denkschrift, http:// www.dfg.de/download/pdf/dfg_im_profil/reden_stellungnahmen/download/ empfehlung_wiss_praxis_1310.pdf (Stand: 23.5.2014).

Duden, 2014 a: Wissenschaft, http://www.duden.de/rechtschreibung/Wissenschaft (Stand: 22.5.2014).

Duden, 2014 b: Plagiat, http://www.duden.de/rechtschreibung/Plagiat (Stand: 22.5.2014)

Fichte, Johann Gottlieb, 2014: Beweis der Unrechtmäßigkeit des Büchernachdrucks. Ein Räsonnement und eine Parabel, http://www.copyrighthistory.com/ fichte.html (Stand: 22.5.2014) (zuerst 1793).

Fishman, Teddi, 2009: „We know it when we see it“ is not Good Enough: Towards a Standard Definition of Plagiarism that Transcendends Theft, Fraud, and Copyright, in: Proceedings oft he Fourth Asia Pacific Conference on Educational Integrity (4APCEI) 28-30 September, University of Wollongong, http:// www.bmartin.cc/pubs/09-4apcei/4apcei-Fishman.pdf (Stand: 22.5.2014).

Franck, Egon, 2005: Die deutsche Promotion als Karrieresprungbrett. Mechanismen der Talentsignalisierung im Ländervergleich, Leipzig.

Hauss, Kalle/Kaulisch, Marc/Zinnbauer, Manuela/Tesch, Jakob/Fräßdorf, Anna/ Hinze, Sybille/Hornbostel, Stefan, 2012: Promovierende im Profil: Wege, Strukturen und Rahmenbedingungen von Promotionen in Deutschland, Ergebnisse aus dem ProFile-Promovierendenpanel, iFQ-Working Paper No. 13, November 2012, http://www.forschungsinfo.de/Publikationen/Download/working_pa per_13_2012.pdf (Stand: 23.5.2014).

Hornbostel, Stefan, 2012: Einleitung, in: Nathalie Huber/Anna Schelling/Stefan Hornbostel (Hrsg.), Der Doktortitel zwischen Status und Qualifikation, iFQ- 
Working Paper No. 12, November 2012, 7-12, http://www.forschungsinfo.de/ Publikationen/Download/working_paper_12_2012.pdf(Stand: 23.5.2014). HRK, 2013: Gute wissenschaftliche Praxis an deutschen Hochschulen, http:// www.hrk.de/uploads/tx_szconvention/Empfehlung_GutewissenschaftlichePraxis_14052013_02.pdf (Stand: 23.5.2014).

KWI, 2014: Essener Thesen, http://www.kulturwissenschaften.de/home/essener thesen.html (Stand: 22.5.2014).

Leggewie, Claus/Mühlleitner, Elke, 2007: Die akademische Hintertreppe. Kleines Lexikon des wissenschaftlichen Kommunizierens, Frankfurt/New York.

Manow, Philip/Flemming, Peter, 2011: Der Titel als politisches Distinktionsmerkmal. Eine Untersuchung akademischer und adeliger Wahlbewerber zum Deutschen Bundestag 1949-2009, in: Zeitschrift für Politikwissenschaft 21 (4), 531-551.

Merton, Robert K., 1983: Auf den Schultern von Riesen. Ein Leitfaden durch das Labyrinth der Gelehrsamkeit, Frankfurt a. M. (3. Aufl.) (zuerst 1965).

New York Times, 1980: Plagiarism Book is Plagiarized, 6. Juni 1980, A28.

Spiegel Online, 2013: Plagiatsvorwurf: Steinmeier bittet Uni um Prüfung seiner

Doktorarbeit, http://www.spiegel.de/unispiegel/studium/plagiatsvorwurf-stein meier-bittet-um-pruefung-seiner-doktorarbeit-a-925281.html

(Stand: 23.5.2014)

Theisohn, Philipp, 2009: Plagiat: Eine unoriginelle Literaturgeschichte, Stuttgart. Weber-Wulff, Debora, 2014: False Feathers. A Perspective on Academic Plagiarism, Heidelberg u. a.

Weiblinger, Julian, 2012: „Plagiat” in der Wissenschaft. Zum Schutz wissenschaftlicher Schriftwerke im Urheber- und Wissenschaftsrecht, Baden-Baden.

Korrespondenzanschrift:

Frederic Hanusch, M. A.

Kulturwissenschaftliches Institut Essen (KWI)

Goethestraße 31

45128 Essen

E-Mail: frederic.hanusch@kwi-nrw.de 